\title{
L'anatomie d'une captation nationaliste : l'absence de mouvements de contestation en Yougoslavie
}

\author{
Natacha Rajakovic
}

\section{(2) OpenEdition \\ 12 Journals}

\section{Édition électronique}

URL : http://journals.openedition.org/conflits/141

DOI : $10.4000 /$ conflits. 141

ISSN : $1777-5345$

Éditeur :

CCLS - Centre d'études sur les conflits lilberté et sécurité, L'Harmattan

Édition imprimée

Date de publication : 21 mai 1992

ISSN : 1157-996X

Référence électronique

Natacha Rajakovic, «L'anatomie d'une captation nationaliste : l'absence de mouvements de contestation en Yougoslavie », Cultures \& Conflits [En ligne], 05 | printemps 1992, mis en ligne le 13 mars 2006, consulté le 30 mars 2021. URL : http://journals.openedition.org/conflits/141 ; DOI : https://doi.org/10.4000/conflits. 141

Ce document a été généré automatiquement le 30 mars 2021.

Creative Commons License 


\title{
L'anatomie d'une captation nationaliste : l'absence de mouvements de contestation en Yougoslavie
}

\author{
Natacha Rajakovic
}

Depuis plus d'un an, l'état yougoslave n'existe plus de facto. Cette réalité s'est trouvée réaffirmée lorsque le 15 janvier 1992, deux des républiques yougoslaves - la Slovénie et la Croatie - ont été reconnues comme états indépendants par la Communauté européenne ainsi que par un grand nombre d'autres pays. L'éclatement de la Yougoslavie a pu sembler d'autant plus surprenant que, depuis le choix par le maréchal Tito en 1948 de construire un socialisme yougoslave "original" qui échapperait à l'emprise de l'Union soviétique, la Fédération yougoslave a été considérée comme l'exemple d'une réussite, au moins partielle, de la réconciliation des modèles politiques et économiques soviétiques et occidentaux d'une part, et des rivalités nationales au sein d'une Fédération pluriethnique d'autre part. Or, la désintégration de l'état yougoslave est due, en grande partie, à l'échec des tentatives successives pour étouffer - au besoin par la coercition - toute possibilité de revendication d'une identité nationale, parallèlement à l'impossibilité de fournir à la société des moyens de contestation qui remplaceraient l'expression des conflits nationaux. La négation de l'existence d'identités nationales distinctes et l'incapacité du pouvoir à fournir des conflits de substitution, conduit précisément au remplacement des divers répertoires de contestation habituellement présents au sein de toute société, par l'unique répertoire que les divers régimes au pouvoir en Yougoslavie depuis 1918 se sont efforcés d'éliminer - et qui, depuis la Seconde Guerre mondiale, a longtemps semblé avoir effectivement disparu - c'est-à-dire le répertoire nationaliste. En effet, la question nationale constitue le facteur qui, plus que tous les autres, conditionne et définit la vie politique de l'état yougoslave depuis sa création en 1918 : l'histoire de la Yougoslavie consiste presque entièrement en une lutte pour résoudre ce problème. 
2 Parce que la question nationale - et par conséquent l'utilisation exclusive du répertoire nationaliste pour exprimer toute contestation de l'ordre étatique - domine et englobe tous les autres conflits politiques ou sociaux potentiels, les mouvements de contestation, tels qu'ils ont existé dans la plupart des autres pays européens, ont été rares en Yougoslavie. Aussi n'a-t-on que très rarement assisté à des mouvements de contestation de masse dans ce pays. Si, depuis la fin des années 1960, des manifestations ou des mouvements de grève sont apparus, ceux-ci étaient le plus souvent l'expression de revendications nationales - sinon nationalistes - malgré l'utilisation, par les initiateurs, de revendications économiques ou politiques "classiques" comme prétextes pour déclencher ces mouvements. Depuis 1990, ce n'est évidemment plus l'ordre politique, ni social ou économique de l'état yougoslave qui est contesté, mais son existence même. Non seulement les républiques et les peuples qui composaient la Yougoslavie ne sont plus satisfaits du régime en place et des autorités au pouvoir, mais ils contestent le bien-fondé de leur appartenance à un état commun. Cette totale remise en cause a pour résultat un obstacle pratique à l'étude des mouvements de contestation : depuis "l'Automne des Peuples" de 1989, on a pu assister à un éclatement progressif de l'état yougoslave, de ses institutions politiques et économiques, de son ordre constitutionnel, et de sa société. On ne peut plus parler aujourd'hui de la Yougoslavie comme entité étatique unique : il faut plutôt considérer chacune de ses républiques séparément, étant donné qu'elles ont chacune leur gouvernement propre, leur constitution, et leur propre dynamique politique, économique et sociale. Parler de l'état ou de la société yougoslaves revient à parler de six états différents. On ne peut donc étudier réellement les mouvements de contestation politique ou sociale à l'échelle yougoslave ou fédérale. De plus, l'éclatement institutionnel, doublé de l'état de guerre dans lequel est plongée la fédération, rend difficile l'accès aux sources nécessaires pour une étude approfondie des émeutes, manifestations, grèves, et autres mouvements de contestation ${ }^{1}$.

3 La logique nationaliste tendant à subordonner tous les autres conflits potentiels au sein de la société yougoslave, elle a constitué, au cours des dernières années, le point culminant de toutes les manifestations et émeutes qui ont eu lieu dans les diverses républiques de la fédération. Depuis 1989, la vie politique, sociale et économique dans ces républiques est en effet caractérisée par la domination de la question nationale sur toutes les autres, tant du côté du pouvoir que du côté de l'opposition. Tous les acteurs qui participent à la vie publique des républiques se servant des mêmes thèmes et de cette même logique nationaliste, l'utilisation d'autres répertoires de contestation devient impossible, puisque le répertoire nationaliste - unique constante au sein de la société yougoslave depuis sa naissance - fusionne tous les autres. La question nationale n'ayant jamais été résolue, mais ayant souvent été niée et étouffée de force par les régimes au pouvoir successifs, elle garde d'autant plus de force comme principale source de contestation par la société. Aussi, pour rallier une partie de la population à un mouvement de contestation (économique, politique ou social) les acteurs politiques se voient obligés de donner à ce mouvement un caractère explicitement national. Le pouvoir et l'opposition étant tous deux confrontés à cette réalité, leur action devient un "jeu à somme nulle", puisqu'on se renvoie des deux côtés les mêmes arguments et que l'on se base sur les mêmes tactiques. Cette crise "par homonymie" des répertoires de contestation et le blocage qui en résulte, rendent impossible tout processus progressif de changement social, économique ou politique, et finit par conduire à un conflit armé entre les républiques. Nous nous efforcerons donc ici de mettre au jour la manière dont 
s'opère la récupération nationaliste de tout mouvement de contestation, au cours de la période qui précède immédiatement le conflit actuel, en prenant pour exemple les manifestations étudiantes de mars 1991 à Belgrade.

5 Afin de mettre au jour les enjeux qui sous-tendent la dynamique du conflit nationaliste contemporain, il est nécessaire de rappeler brièvement l'histoire des modes de gestion des mouvements de contestation en Yougoslavie depuis 1945. Tel qu'il a existé jusqu'aux années 1980, le système sociopolitique yougoslave avait ceci de particulier qu'on pouvait difficilement l'insérer dans une quelconque classification des pays du monde contemporain. A partir de 1945, la Yougoslavie passe en effet rapidement d'un extrême à l'autre. D'un engagement presque total de la société dans un double conflit (contre l'agresseur externe d'une part, entre les nations qui composent le pays de l'autre) au cours de la deuxième guerre mondiale, on passe à une société, du moins en apparence, sans conflits ${ }^{2}$. Puis de ce semblant d'harmonie sociale et politique de l'après-guerre, on arrive, à la fin des années 1980, à une situation de conflit telle, qu'elle conduit l'état à sa désintégration. La question nationale relie les deux périodes car, dans les deux cas, elle se trouve être à la base même de l'engagement de la société dans le conflit. Après l'écroulement du "premier" état yougoslave en 1941, la majorité de la population se trouve, dans un premier temps, mobilisée pour défendre les intérêts de telle ou telle nation particulière à laquelle on appartient. Il en résulte une guerre "civile" qui compte parmi les plus meurtrières de l'Histoire moderne. Dans un deuxième temps, le Mouvement de libération nationale mobilise jusqu'aux populations les moins politisées contre l'ennemi extérieur, pour défendre la "nation" yougoslave. Le succès de cette mobilisation repose sur deux facteurs: d'une part, toutes les nations yougoslaves se trouvant agressées et occupées sans distinction, la lutte contre l'ennemi a pour effet de restaurer entre elles "l'égalité", alors qu'elles s'étaient toutes senties "flouées" sur le plan politique ou économique - souvent les deux au sein du premier état yougoslave. D'autre part, l'égalité retrouvée permet l'organisation d'une Résistance commune qui efface du moins pour un temps - les conflits nationaux, et permet au régime qui s'installe au pouvoir de justifier non seulement la réconciliation nationale mais également la disparition - soi-disant définitive - de la question nationale. En effet, puisque Serbes, Croates, Slovènes, Monténégrins et autres ont combattu côte à côte, cela signifie qu'une vie commune est possible et que les conflits qui les avaient opposés au cours des décennies précédentes étaient dus à la nature du régime politique "corrompu" (la monarchie bourgeoise) auquel ils étaient soumis ainsi qu'à des influences extérieures négatives. Le programme de "révolution sociale" qui accompagne le Mouvement de libération nationale renforce les fondements du nouveau système politique marxiste instauré par le maréchal Tito et par ses partisans à l'issue de la guerre. Quelques années plus tard, l'engagement sollicité par la nouvelle confrontation avec un ennemi extérieur (Staline), consolide définitivement les assises du régime titiste. Ce conflit continu avec des agresseurs externes réels ou potentiels, qui se prolonge jusqu'à la fin des années 1940, mobilise suffisamment la société yougoslave pour commencer à subordonner les conflits internes potentiels. L'organisation politique et sociale de type marxiste achève d'étouffer toutes les velléités de revendication politique, économique ou sociale au sein de la société. Le pouvoir se trouve confirmé dans son rôle de représentant unique et véritable de "l'intérêt national", grâce à son identification à un triple symbole déifié: Tito/la Résistance/le Parti communiste, auquel le reste de la société est également fortement 
"encouragé" à s'identifier. Le fait que ce triple pouvoir assure la sécurité et l'identité nationales face au danger externe; qu'il annonce un programme de transformation sociale ; et qu'il dispose de moyens de pression concrets sur la société civile (utilisation de méthodes coercitives semblables à celles en vigueur dans les autres états autoritaires du bloc communiste) renforce ses assises. Dès la fin des années 1940 et jusqu'à la moitié des années 1960, la coercition jouera un rôle de plus en plus important. A mesure que s'éloigne et faiblit le mythe de la Résistance, le régime accroît son utilisation de la force et des mesures punitives pour enrayer toute velléité de contestation, nationale ou autre.

6 Au cours des années 1950-1960, la société yougoslave approche de près, dans le discours idéologique du pouvoir, le modèle de la société sans conflits. Cette société est surtout caractérisée par : 1 - Le "collectivisme", ou l'attachement à la primauté des intérêts communs à toute la société ("l'intérêt général"). L'intérêt général étant déterminé à l'avance par le Parti communiste, la sphère de participation de l'individu dans la vie publique est limitée à l'expression formelle et symbolique de son attachement au système. Dans ces conditions, les conflits et revendications potentiels sont refoulés au profit de ce que l'on croit être le bien collectif, et toute contestation ouverte de l'ordre établi devient difficile. 2 - La valeur majorée du concept d'égalité, qui doit servir à réduire au maximum la distance entre divers groupes sociaux et exclut toute possibilité de compétition réelle entre ces mêmes groupes et entre les individus. La suppression en apparence des antagonismes de classe, contribue à renforcer l'idéal d'une société égalitaire. L'égalité proclamée occulte en réalité l'apparition de nouvelles disparités de classe entre l'élite dirigeante et les membres du Parti communiste d'une part, le reste de la société d'autre part. En Yougoslavie, de fortes inégalités régionales se développent également, notamment entre les républiques septentrionales, qui jouent le rôle de "moteur" économique et les républiques méridionales, dont le retard économique s'accentue et les place dans une relation de dépendance vis-à-vis des premières. 3 L'harmonie sociale, qui constitue un des fondements de la société marxiste. La collaboration entre les individus est non seulement un des moyens pour atteindre un état d'harmonie mais un but en soi.

7 Ces caractéristiques, qui favorisent l'uniformisation en surface de la société et la passivité de 1 individu et des groupes en tant qu'acteurs sociaux - ils comptent sur le Parti pour assurer leur sécurité sociale et la récompense morale qui leur est promise empêchent les conflits potentiels d'être reconnus et exprimés au sein de la société. De plus, toute tentative d'exprimer une quelconque différenciation est interprétée comme preuve irréfutable d'hostilité au régime et à l'intérêt collectif Ceux qui s'aventurent dans cette voie sont immédiatement désignés comme ennemis - de classe, de la nation, de l'état, etc. - et menacés d'exclusion. Aucun acteur politique ne peut donc prendre le risque de s'aventurer hors du cadre imposé par l'état marxiste. L'intolérance envers la différenciation au sein de la société, ainsi que les structures répressives, rendent donc pratiquement impossibles les manifestations grèves, et autres moyens d'expression de la contestation politique. Au cours des années 1960, la Yougoslavie s'ouvre progressivement à l'Occident. Soucieux d'assurer à son état un rôle clef sur la scène internationale, le maréchal '15to ouvre les frontières autorise 1 importation de biens de consommation, ce qui entraîne l'introduction, dans la société yougoslave, de valeurs traditionnellement associées au système capitaliste (le tourisme joue également un rôle important dans l'ouverture de la société yougoslave vers l'Occident). L'autogestion, qui, dans une certaine mesure, libéralise l'économie, contribue à une plus grande souplesse 
dans l'organisation sociale et à une réorientation des valeurs. Si l'individualisme n'est pas encore reconnu expressément comme étant l'une d'elles le climat de libéralisation encourage certains groupes, tels les étudiants et les intellectuels, à s'aventurer, à exprimer de plus en plus ouvertement leur insatisfaction concernant l'ordre économique, puis politique, établi au lendemain de la guerre. En outre, l'apparition de l'insécurité sociale, avec l'introduction de certains éléments de "l'économie de marché", l'accroissement du chômage, l'émigration des travailleurs yougoslaves, qui conduit à la confrontation des buts révolutionnaires professés au lendemain de la guerre avec les conditions sociales réelles vingt ans plus tard, amène un certain nombre d'individus à remettre en question le système, du moins dans son application pratique. La société yougoslave connaît, pour la première fois depuis 1948, une situation de conflit ouvert, au cours des manifestations étudiantes dans les grandes villes, à Belgrade d'abord, puis à Ljubljana et à Zagreb, entre 1968 et $1971^{3}$. Ce climat de libéralisation relatif à la fin des années 1960 permet à la question nationale, étouffée de force et par en haut depuis la fin de la guerre, de redevenir l'enjeu central des conflits au sein de la société et de la classe dirigeante yougoslaves.

8 Les manifestations étudiantes qui ont lieu en juin 1968 prennent une forme "classique", dans la mesure où elles consistent en l'organisation de meetings sur les campus universitaires et en la signature de pétitions en faveur de l'élimination des inégalités sociales et du chômage, de la démocratisation politique, de l'amélioration du statut des étudiants et d'une plus grande participation des étudiants dans les processus décisionnels visant à résoudre les conflits sociaux. Cependant, ces manifestations sont de courte durée (deux semaines) et se limitent aux grands centres universitaires. Aucune ne se transforme en émeute, leur ampleur ne dépassant pas les limites que l'on pense pouvoir être tolérées par le régime en place.

9 Les deux premiers mouvements de contestation politique significatifs par leur ampleur et les conséquences politiques qu'ils entraînent, sont la manifestation de masse au Kosovo en novembre 1968 et le "Printemps Croate" de 1971. Au cours de cette période, la lutte politique sous une bannière ouvertement nationaliste n'est pas encore possible. Il est donc nécessaire de lui donner une apparence idéologique. Si les manifestations des étudiants albanais du Kosovo et le mouvement des intellectuels croates comportent, dès le départ, des éléments de revendication nationale, ils se présentent officiellement d'abord comme des mouvements en faveur de la libéralisation économique. En effet, à partir du 27 novembre 1968, les étudiants kosovars manifestent en masse pour réclamer une meilleure distribution des ressources entre les républiques, et en profitent pour introduire l'idée de la transformation de leur province en république, pour lui donner le même statut que les six autres qui constituent la fédération yougoslave.

10 Dans un schéma semblable, la théorie de "l'autogestion" joue, à partir des années 1965-66, pour les intellectuels croates et pour un bon nombre de dirigeants de la Ligue des communistes de Croatie un rôle capital dans l'élaboration des méthodes de contestation politique. En effet, puisque l'autogestion représente l'innovation la plus spectaculaire du communisme yougoslave, il est toléré, au sein de la Ligue des communistes, de contester en son nom le centralisme unitariste. Or, en transférant les capitaux du niveau fédéral vers les sociétés autogestionnaires, on les transfère par la même occasion aux républiques. Ceci permet de développer, sous la bannière du libéralisme économique, un répertoire de revendications nationalistes. Ainsi naît, à 
partir de 1970, un mouvement visant à obtenir la reconnaissance formelle de la spécificité culturelle croate et un transfert d'un certain nombre de responsabilités politiques, économiques et sociales du niveau fédéral vers celui de la république. Le caractère "nationaliste" du mouvement apparaît dès les premières manifestations étudiantes à Zagreb, en novembre 1971 en effet, un certain nombre de manifestants se posent ouvertement en "défenseurs" de la renaissance culturelle et politique croate, menacée selon eux par les manifestants serbes de Belgrade. Ces deux mouvements de contestation constituent la première crise politique grave de la Yougoslavie communiste, qui remet en question l'organisation et la survie de l'état. A partir de 1971, le spectre de la désintégration de la fédération ne quitte plus l'esprit des dirigeants yougoslaves. Si la répression est sévère dans les deux cas (en Croatie surtout, il y aura plus de 400 arrestations au cours des mois qui suivent les manifestations, tous les organisateurs du mouvement sont condamnés à des peines de prison de plusieurs années, certains forcés à un exil qui ne s'achève qu'en 1990, et la direction du Parti communiste croate est entièrement "purgée" de tous les éléments jugés "nationalistes" ou trop indulgents à Cette contestation ouverte de l'ordre l'égard des manifestants) politique yougoslave, exprimée lors de manifestations populaires, conduit cependant, en 1974, à l'élaboration d'une nouvelle constitution, destinée à renforcer la cohésion nationale de l'état (cette Constitution représente l'ultime tentative, par le régime titiste, de résoudre définitivement la question nationale.

11 La sévérité de la répression des mouvements des années 1968-1971 empêche toute tentative de contestation politique ouverte et massive au cours de la décennie suivante. Ce n'est qu'en 1981 qu'un nouveau mouvement de masse apparait, une fois encore défini par la revendication nationale. En effet, en mars 1981, les étudiants de l'université de Pristina (capitale de la Province autonome du Kosovo) manifestent pour l'obtention de meilleures conditions de logement et contre l'accroissement du chômage parmi les jeunes diplômés. Si les autorités semblent garder le contrôle de la situation au départ à partir du 26 mars, les manifestations commencent à s'étendre avec la participation d'un grand nombre d'ouvriers. A partir de ce moment, le mouvement prend un caractère ouvertement nationaliste et les revendications tournent autour $\mathrm{du}$ statut de république pour le Kosovo et de la reconnaissance formelle de la spécificité culturelle de la population albanaise. Les affrontements entre les manifestants et la police se soldent par des dizaines de morts et plusieurs centaines de blessés. Des troupes de l'armée fédérale sont envoyées en renfort, l'état d'urgence est décrété et le Kosovo isolé du reste du pays. Au cours des semaines et des mois suivants, le Parti communiste, le gouvernement et l'administration locale du Kosovo sont entièrement purgés. Les procès contre les organisateurs et les participants des manifestations se poursuivirent jusqu'en 1982-1983, quelque 1500 personnes sont condamnées pour crimes politiques, 4500 pour autres délits et les condamnations vont jusqu'à quinze ans de réclusion. Ces émeutes et la répression qui s'ensuivent, menée par les autorités serbes avec le concours de l'armée fédérale, ont conduit certains Yougoslaves à utiliser le terme de "bataille du Kosovo" pour évoquer ces événements, par référence à la mythique bataille de Kosovo de $1389^{4}$.

12 Les émeutes de 1981 marquent en réalité la réapparition en force de la question nationale, qui à partir de ce moment, devient sans conteste l'unique agent "mobilisateur" en Yougoslavie. Du côté des autorités serbes à Belgrade, comme du côté des manifestants, l'élément majeur du débat politique est la menace que constitue chacune des communautés pour l'existence et la survie de l'autre. En effet, les Serbes 
orthodoxes, qui de 23,6 \% de la population du Kosovo en 1961 ne constituent plus que $13,2 \%$ en 1981, se sentent menacés par une population albanaise et musulmane en pleine expansion. Les Albanais, de leur côté, ne pardonnent pas à la minorité serbe sa position privilégiée dans les domaines politique, administratif et dans celui des services.

Les émeutes de 1981 au Kosovo coïncident avec la première année de la période posttitiste et marquent le début du processus de désintégration de l'état yougoslave. Au moment où, dans un certain nombre de pays d'Europe centrale et orientale, la carapace communiste commence à se fissurer et les populations descendent dans la rue pour réclamer la libéralisation du système politique et économique, en Yougoslavie, les mouvements de contestation deviennent autant de signes concrets de l'absence d'une véritable nation yougoslave. En effet, on peut constater que des manifestations et des grèves sont parfois organisées au même moment dans différentes républiques de la fédération, mais elles n'ont jamais la même origine, ni les mêmes buts. Tous les mouvements de contestation sociale ou politique sont des mouvements "nationaux" et non "fédéraux". Par ailleurs, on peut noter que, malgré la violence qui accompagne les mouvements de contestation de 1968-1971, de 1981 et même ceux de la fin des années 1980, on ne peut réellement parler "d'émeutes". En effet, la violence fait toujours son apparition au moment de l'intervention des forces armées et de la police. Si des actes de provocations isolés peuvent certes avoir lieu dans les rangs des manifestants et des grévistes, les mouvements ne sont jamais accompagnés d'actes de vandalisme organisés et systématiques par les manifestants et on ne constate jamais la présence de groupes extérieurs aux mouvements eux-mêmes, qui pourraient conduire à une "dérive" violente des manifestations. La violence demeure toujours le monopole presque exclusif des autorités fédérales et des unités spéciales de la police, qui agissent à la demande du pouvoir politique central.

La fragilité de la fédération yougoslave commence à apparaître de manière spectaculaire dès 1987. A partir de cette période, deux phénomènes indissociables se développent parallèlement: d'une part, une montée rapide des nationalismes, déclenchée par l'arrivée au pouvoir, en Serbie, du leader communiste-populiste Slobodan Milosevic. D'autre part, un nombre impressionnant de grèves et de manifestations ouvrières marque la fin des années 1980 et indique clairement le degré avancé de décomposition du système économique, monétaire et institutionnel de la fédération yougoslave. En 1987, on compte 1623 grèves, soit quelque 365000 grévistes et 1720 grèves en 1988, soit 400000 grévistes. La plupart sont organisées dans le but professé d'obtenir des conditions salariales meilleures, mais servent souvent à "dénoncer" l'exploitation économique d'une des républiques par les autres et par le centre.

15 A partir de l'automne 1988, la scène politique yougoslave est marquée par la jonction définitive entre les grèves revendicatives de type "classique" et les manifestations politiques, sous la houlette des dirigeants serbes. Ceux-ci se battent pour un renforcement des pouvoirs centraux de la fédération et s'opposent à toute libéralisation au sein de la Ligue des communistes yougoslaves. Pour assurer les assises de son pouvoir, Milosevic organise une campagne de rassemblements de solidarité avec la population serbe et monténégrine du Kosovo. Le dénominateur commun de ces meetings est de réclamer un changement de la Constitution yougoslave de 1974 qui permettrait à la Serbie de recouvrer ses pleines prérogatives sur ses deux provinces 
autonomes de Voïvodine et du Kosovo - ce qui sera fait en mars 1989 grâce à un amendement constitutionnel. Les slogans scandés par les manifestants de Milosevic ont un caractère incontestablement nationaliste : "Donnez-nous des armes!", "Nous irons au Kosovo où ils tuent nos frères!" sont des phrases que l'on retrouve dans toutes les manifestations des années 1989-1990. Ces "ralliés" se déploient en Serbie et dans les régions à dominante serbe ou proches par l'histoire (Monténégro, Voïvodine). Aux revendications en apparence économiques et sociales s'ajoutent des thèmes politiques, tels que le soutien des Slaves "menacés de génocide" par les Albanais, la dénonciation de la "trahison" subie par la Serbie sous le régime titiste - accusations de détournements des capitaux de la Serbie vers la Croatie et la Slovénie et d'exploitation économique et politique par les républiques septentrionales - ainsi qu'une campagne en faveur de la "révolution anti-bureaucratique" menée par le nouveau leader serbe. Le caractère nationaliste des manifestations organisées par Milosevic devient explicite lors du rassemblement à Kosovo Poljé en juin 1989, qui marque le 600ème anniversaire de la bataille du Kosovo: dans son discours, Milosevic affirme que les nouveaux dirigeants serbes ont "réunifié" la Serbie, que celle-ci était désormais engagée dans une bataille et qu'un conflit armé ne peut être exclu. Au Kosovo même pour contrer le mouvement nationaliste serbe et la "normalisation" imposée à la province, des manifestations, des grèves générales et des grèves de la faim sont organisées de nouveau. Les manifestations les plus violentes de l'année 1989 - et en fait depuis la Seconde Guerre mondiale - ont lieu en février-mars 1989, dans le but de protester contre la mainmise serbe sur la Voïvodine et le Kosovo, et contre l'arrestation d'Azem Vllasi, ex-président du Parti communiste du Kosovo. Ces manifestations débutent par une grève (y compris une grève de la faim) des mineurs de Trepca le 21 février 1989 rapidement transformée en grève générale au Kosovo - qui exigent la démission de dirigeants albanais mis en place par la direction du Parti communiste de Serbie. Des manifestations organisées dans plusieurs grandes villes conduisent à l'affrontement direct entre manifestants et forces de la police. Comme en 1981, les autorités fédérales réagissent par l'envoi de forces armées spéciales (10 000 soldats pour le seul mois de février 1989), suivi de nombreuses arrestations et d'un couvre-feu sur l'ensemble du Kosovo. Le couple manifestation/répression provoque plusieurs dizaines de morts et de blessés. Les dirigeants des républiques croate et slovène dénoncent alors pour la première fois l'association trop étroite entre les revendications économiques et les revendications politiques qui comporte, selon eux, un danger inhérent de radicalisation. En effet, cette association, permet à la Ligue des communistes de Serbie d'apparaitre comme le rempart des Serbes contre une politique qui se mène sans eux et même contre eux, et facilite la revanche du nationalisme serbe sur la ligne politique de certains membres des Ligues slovène et croate, pour lesquels une amélioration sensible de la crise yougoslave passe nécessairement par une libéralisation politique en profondeur.

16 La montée du nationalisme grand-serbe inspiré par Milosevic provoque, à partir de 1989, le développement de mouvements de réaction nationalistes slovènes et croates, dont les dirigeants sont rapidement entraînés dans un jeu de "surenchère". L'opposition politique serbe, qui commence à s'organiser à cette époque, est également obligée d'avoir recours aux sentiments nationalistes si elle désire faire face à la politique populiste de Milosevic. Ainsi, toutes les manifestations de mécontentement populaire, à partir de ce moment, seront définies exclusivement par le thème de 
l'appartenance à une nation et de la sauvegarde des spécificités et des privilèges de celle-ci au sein de l'état yougoslave. la désintégration de la Ligue des communistes de Yougoslavie en janvier, les institutions fédérales sont les unes après les autres, progressivement affaiblies et contestées, par les dirigeants des républiques. Des élections pluralistes sont organisées, à la suite de campagnes électorales où dominent des revendications nationales: d'abord en Slovénie et en Croatie (avril-mai 1990), puis en Bosnie-Herzégovine (novembre 1990), et enfin en Macédoine, en Serbie et au Monténégro (décembre 1990). $\mathrm{Au}$ cours de ces élections, on ne vote pas réellement pour de nouveaux programmes politiques, mais plutôt contre le régime communiste, et surtout contre les dirigeants d'une autre république. Partout, à l'exception de la Serbie et du Monténégro voisin, le pouvoir communiste est remplacé par des autorités issues de l'opposition. De nouvelles constitutions sont adoptées par les républiques, qui donnent à leur législation la primauté par rapport à la législation fédérale, y compris au Kosovo, où une nouvelle constitution proclame le gouvernement par la majorité, l'autodétermination et l'indépendance de la république du Kosovo. Seule parmi les institutions fédérales, l'armée tente de préserver son caractère yougoslave, ses privilèges et son prestige. L'affrontement national entre les républiques du Nord d'une part, engagées sur la voie de la démocratisation politique et de la libéralisation économique, qui souhaitent transformer l'état yougoslave en une association souple d'états souverains, tout en refusant le maintien de l'armée dans le débat politique, et la Serbie et le Monténégro d'autre part, qui souhaitent, au contraire, un état plus centralisé, un gouvernement fédéral fort et le maintien de l'armée comme arbitre politique, s'aggrave rapidement.

Les relations s'avèrent particulièrement tendues entre les républiques slovène et croate d'une part, et serbe d'autre part, à partir de l'été 1990. L'agitation nationaliste de la minorité serbe de Croatie ( $12 \%$ de la population totale de la Croatie), le plus souvent organisée et soutenue par Milosevic et ses partisans, exacerbe les sentiments nationalistes croates. Une "guerre des nerfs" est engagée entre la Croatie et la Serbie à partir d'août 1990, lorsque la population serbe de Croatie, au cours d'un référendum, vote en masse pour une "autonomie culturelle et politique" des provinces où elle se trouve concentrée. Les incidents se multiplient dès lors entre les communautés croate et serbe, puis se transforment progressivement pour exploser en guerre ouverte. Paradoxalement, un des événements majeurs qui déclenche le conflit ouvert en mars 1991, tant à l'intérieur de la Serbie même qu'entre les républiques, est l'unique manifestation de masse - accompagnée d'actes de violence non seulement par les forces de l'ordre mais également parmi les manifestants - de l'Histoire yougoslave récente, qui incarne une véritable tentative de contestation de l'ordre politique et une volonté de démocratisation par l'opposition serbe.

Le mécontentement à l'égard de la politique du président serbe avait commencé à se manifester au cours des derniers mois de 1989, en particulier au sein des milieux intellectuels, d'affaires et étudiants. Ces derniers reprochent aux dirigeants serbes en général, et à Milosevic en particulier, de ne pas remplir les promesses répétées depuis son élection en 1987. La crise économique s'aggrave en effet, malgré les assurances données sans arrêt par Milosevic d'orienter l'économie vers des structures de marché. Entre décembre 1989 et décembre 1990, cinquante-deux partis d'opposition sont créés. 
Cependant, malgré, et peut-être à cause de leur nombre, ces partis ne parviennent ni à s'organiser de manière à rendre leur action efficace, ni à participer à l'élaboration de la nouvelle loi électorale. Ils sont entièrement mis à l'écart par les autorités au pouvoir, l'accès aux médias, dirigés par des membres du Parti communiste, fidèles à Milosevic, leur étant presque entièrement défendu. Ne pouvant pas réellement participer à la campagne électorale, l'Opposition menace jusqu'au dernier moment de boycotter les élections et ne parvient finalement à obtenir que des résultats médiocres. Aussi, on assiste en Serbie, au début de l'année 1991, à un phénomène semblable à celui qui s'est produit en Roumanie et en Bulgarie : le lendemain des élections, l'opposition, soutenue de plus en plus ouvertement par certains segments de la population, en conteste le résultat. A partir de mars 1991, Milosevic se trouve face à une situation en apparence paradoxale : plébiscité trois mois plus tôt au cours des premières élections pluralistes de l'après-guerre, il voit son pouvoir sévèrement remis en cause au cours de manifestations étudiantes en mars 1991. Le samedi 9 mars, la triple crise économique, politique et nationale mobilise, pour la première fois, la jeunesse serbe. Ce jour-là et les jours qui suivent, la capitale fédérale et serbe, Belgrade, connaît une explosion des "ranc urs", accumulées au cours des années précédentes. La Serbie, en quête d'une nouvelle identité au sein de la fédération éclatée exprime enfin ouvertement toutes les contradictions internes qui divisent les dirigeants centralistes, auréolés d'un verdict électoral contesté par leurs adversaires et une opposition grandissante, déchirée entre son nationalisme - aussi puissant que celui des autorités communistes - et un désir de démocratisation qui la rapproche des expériences en cours en Slovénie et en Croatie.

21 La manifestation du 9 mars, visant à dénoncer le manque d'objectivité des médias serbes, et en particulier de la télévision de Belgrade est annoncée par le slogan : "Nous libérons la télé-Bastille !". Dans un premier temps, malgré l'interdiction de manifester annoncée le 7 mars par le ministère de l'Intérieur de Serbie, près de 100000 personnes - dont le gros est composé de jeunes (chômeurs et étudiants confondus) - se rassemblent au centre de Belgrade, à l'appel de dix partis d'opposition, notamment du Parti du renouveau serbe, dirigé par un des opposants les plus acharnés de Milosevic, l'écrivain nationaliste Vuk Draskovic. Les manifestants réclament "l'autonomie de l'information" et protestent contre la mainmise des autorités communistes de Serbie sur les médias, en demandant notamment la démission de cinq dirigeants de la télévision, la création d'une chaîne "apolitique" et le droit pour les télévisions indépendantes d'utiliser les émetteurs du réseau officiel. Cependant, quelques heures avant le début programmé de la manifestation, les autorités de Serbie ont décidé de devancer les manifestants : un important dispositif policier, déterminé à faire respecter l'interdiction de manifester, barre l'accès au lieu fixé du rassemblement. Des contrôles sont effectués à l'entrée de la ville, afin d'empêcher la participation des manifestants venus de province. Quinze minutes avant le début officiel de la manifestation, la police actionne les canons à eau et lance les premiers gaz lacrymogènes. Dans un discours passionné, Vuk Draskovic lance "A l'assaut!", appel qui marque le début de l'affrontement direct entre manifestants et forces de l'ordre.

Dans un deuxième temps, la manifestation se transforme en rébellion anticommuniste, réclamant la démission des principaux dirigeants, dont Slobodan Milosevic lui-même. Les manifestants scandent des slogans tels que "Slobo-Saddam", "Slobo-Staline", "A bas la Mafia rouge". La tentative, par les manifestants, d'approcher certains bâtiments officiels et d'enfoncer le cordon de police provoque une réaction plus violente encore des forces de l'ordre et la manifestation se transforme en "émeute", accompagnée pour 
la première fois d'actes de vandalisme par des "casseurs". Le bilan des affrontements est de deux morts et plus de cent blessés. Les collaborateurs de Milosevic saisissent la présidence fédérale, dirigée à cette époque par le serbe Borislav Jovic. En dépit de l'avis négatif des représentants de la Slovénie et de la Croatie, qui ne veulent pas voir l'armée affectée à des tâches de police, la présidence opte pour l'utilisation des forces armées et l'envoi des chars dans les rues de Belgrade ${ }^{5}$. Vuk Draskovic et deux de ses adjoints sont arrêtés. Pour montrer leur détermination à ne pas céder, les autorités serbes font fermer les stations de télévision et de radio indépendantes, et des perquisitions ont lieu dans les locaux des journaux non contrôlés par le pouvoir. Le procureur de la république de Serbie interdit "toute information pouvant troubler l'ordre public", et applique une censure totale de l'information concernant la manifestation et les émeutes qui s'ensuivent. Ainsi les Belgradois ignorent que le dimanche 10 mars, plusieurs milliers d'étudiants continuent de manifester à la cité universitaire, exigeant la démission immédiate du ministre de l'Intérieur de Serbie et la libération des dirigeants de l'opposition et des manifestants arrêtés. Dix-neuf députés du Mouvement du renouveau serbe entament une grève de la faim dans les locaux du Parlement de Serbie, alors que le Parti démocrate retire ses députés du Parlement. Au cours des jours suivants, les manifestations et les affrontements entre étudiants et forces de l'ordre beaucoup plus limités dans leur ampleur - se poursuivent en alternance avec des négociations entre opposants et membres du gouvernement serbe. Le lundi 11 mars, le parti de Milosevic, surpris par l'ampleur de la contestation, décide de contrer le mouvement en mobilisant quelque 100000 personnes de province, qui "manifestent" pour vilipender les "fascistes" et les "assassins", alors que les "mères ouvrières "pleurent sur l'opposition qui "embrigade nos enfants". Le même jour ont lieu de nouveaux affrontements entre manifestants et forces de l'ordre à Belgrade. Néanmoins, Milosevic et les autorités serbes sont obligés d'accorder des concessions de surface, d'autant plus que les manifestations s'étendent aux universités de province et que des institutions telles que la prestigieuse Académie des Sciences de Serbie, qui avait vu en Milosevic un défenseur de la cause serbe, se rallient aux étudiants. Dès le 12 mars, Vuk Draskovic est libéré, de même que tous les manifestants arrêtés lors de la manifestation du 9 mars. Le directeur de la télévision est obligé de démissionner, ainsi que quatre de ses collaborateurs et une commission d'enquête parlementaire, chargée de déterminer les responsabilités dans les troubles, est créée. Le lendemain, c'est le ministre de l'Intérieur, Hadmilo Bogdanovic, qui se voit obligé de présenter sa démission. Après cinq jours de manifestations, les étudiants qui occupent le centre de Belgrade, se dispersent enfin à l'appel de députés démocrates. Cependant, comme le déclare un des étudiants : "Plus rien ne sera comme avant"'.

23 En effet, les manifestations de début mars enclenchent la dynamique de déstabilisation finale, non seulement au sein de la Serbie même, mais également dans toute la fédération. En outre, les émeutes de Belgrade marquent le début effectif de la désintégration des institutions politiques fédérales et de l'affrontement direct entre Milosevic et les autorités serbes d'une part, et les autorités des autres républiques d'autre part. Réalisant que son pouvoir est pour la première fois sérieusement menacé, Milosevic choisit la "fuite en avant" et la stratégie du pire. Au cours des jours qui suivent les grandes manifestations des 9-11 mars, Milosevic et ses alliés provoquent un blocage constitutionnel : le représentant de la Serbie au sein de la présidence, Borislav Jovic, démissionne, ainsi que ses alliés, les représentants du Monténégro et de la Voïvodine. Milosevic déclare que "la Yougoslavie est entrée dans la phase finale de son 
agonie" et que désormais, il ne reconnaît plus aucune légitimité à sa présidence. De plus, il annonce qu'il entend démettre le représentant de la Province du Kosovo, qui avait voté contre les mesures d'urgence. Réduite ainsi à quatre membres uniquement (Slovénie, Croatie, Bosnie-Herzégovine et Macédoine), la présidence collégiale n'atteint pas le quota nécessaire pour valider ses décisions et le fonctionnement des plus hautes instances fédérales est bloqué. I1 apparaît simultanément que la lutte menée par Milosevic contre l'opposition, est indissociable de la lutte nationaliste qui l'oppose aux républiques septentrionales. En effet, le jour même de son discours annonçant la mort de la Yougoslavie, les dirigeants serbes de la région de Knin (sud-ouest de la Croatie) annoncent leur détachement de la république croate et se constituent en "Région autonome serbe", événement qui marque le véritable début de la guerre ouverte entre les républiques serbe et croate. Lorsque Milosevic arrive au pouvoir en 1987, l'opinion publique serbe se caractérise par le ressentiment profond quelle éprouve pour Tito : en effet, elle ne lui pardonne pas d'avoir soustrait les deux provinces du Kosovo et de la Voïvodine à l'emprise directe de la Serbie. En exploitant ce sentiment, Milosevic s'assure rapidement un soutien massif. La popularité du président serbe vient du fait qu'il parvient à redonner à la population serbe la "conscience nationale" étouffée par des décennies de communisme "titiste", qui base l'équilibre de la fédération yougoslave sur la position "affaiblie" de la Serbie. Au printemps 1987, au cours du rassemblement de Kosovo Poljé, Milosevic prononce une phrase clef: Nul n'a le droit de battre ce peuple (1e peuple serbe nda.)", déclare-t il en réponse aux revendications de la minorité serbe du Kosovo, qui se plaint d'être en proie à l'hostilité de la majorité albanaise. Le succès évident de ces premières campagnes, basées presque exclusivement sur le thème de la "reconquête" des deux provinces autonomes, encourage donc le président serbe à continuer de s'appuyer sur des arguments nationalistes, même après avoir effectivement réintégré le Kosovo et la Voïvodine à la Serbie en 1989. Ainsi, lorsque les dirigeants communistes sont écartés du pouvoir les uns après les autres au cours des élections de 1990, Milosevic craint que la Ligue des communistes de Serbie (rebaptisée Parti socialiste), ne connaisse le même sort. I1 décide donc, une fois encore, de se servir du répertoire nationaliste. En tentant d'imposer un blocus économique à la Slovénie, en incitant et en appuyant les revendications nationalistes de la minorité serbe en Croatie et en empêchant l'opposition serbe de participer pleinement à la campagne électorale, il assure le succès de son parti en décembre 1990.

Cependant, alors que Milosevic parvient facilement à maintenir un état de "conflit ethnique" permanent entre la population serbe et les républiques voisines à partir de 1989, son incapacité à introduire les réformes économiques promises et à améliorer le niveau de vie en Serbie même devient rapidement évidente. Cet échec économique, ajouté au contrôle quasi-absolu des médias serbes par le gouvernement et au refus de toute négociation avec les partis d'opposition est à l'origine des manifestations du 9-11 mars 1991. Les manifestants commencement par réclamer la fin de la "Milosevision", accusée "d'attiser les passions nationalistes et de pousser les Serbes à la guerre civile". En effet, à travers son contrôle des médias, Milosevic favorise le développement d'une hostilité de plus en plus aiguë envers les nations "rivales" de la Serbie. Ainsi, le journal Politika Ekspres décrit quotidiennement les Croates comme "des fascistes à la solde du Vatican, décidés à mener un génocide contre le peuple serbe". Par conséquent, si le mythe de l'unité serbe s'évanouit à ce moment, les manifestations ont pour résultat de renforcer, au sein de l'opposition serbe, comme au sein du gouvernement, l'importance 
de la question nationale. Milosevic est le premier homme politique serbe de la période récente à comprendre que pour survivre, son parti se doit de devenir le héraut d'un sentiment nationaliste débordant au sein de la population, sentiment qui ne demande qu'à s'exprimer. Ce faisant, il facilite l'apparition et le succès d'autres "hérauts" du nationalisme. En réalité, dès 1990, une partie de l'opposition serbe, notamment le plus puissant - le Parti du renouveau serbe - est caractérisée par un militantisme nationaliste aussi virulent que celui de Milosevic. Alors que le Président serbe soutient le maintien d'une Yougoslavie unie, où la Serbie occuperait une position prépondérante, Vuk Draskovic, l'organisateur des manifestations de mars 1991, se fait l'avocat de la dissolution de l'étai yougoslave et de la création d'une "Grande Serbie".

Les slogans utilisés au cours des manifestations de mars 1991 démontrent l'importance du nationalisme comme unique moyen de mobilisation efficace pour le pouvoir comme pour l'opposition serbes. Dès le lundi 11 mars, les manifestants de Belgrade scandent "Serbie! Serbie!" exclamation reprise au cours de la contre-manifestation organisée par le Parti socialiste de Milosevic. Au cours de cette dernière, les organisateurs de la manifestation du 9 mars sont par ailleurs traités de "traîtres". Le même jour, lorsque le patriarche Paul, de l'église orthodoxe serbe, demande aux manifestants de se disperser, "dans l'intérêt de l'unité serbe il est hué et traité lui aussi de "traître". Lorsque Milosevic s'adresse au Parlement serbe, il déclare que "seuls les ennemis de la Serbie peuvent aspirer à déstabiliser la république, afin qu'elle soit plus faible dans le débat sur l'avenir de la Yougoslavie". Ainsi, il s'agit pour l'opposition comme pour le pouvoir, de désigner clairement les défenseurs véritables de la Serbie $d$ une part, les "traîtres" d'autre part - le côté opposé n'étant pas uniquement considéré comme un adversaire politique, mais également comme un ennemi de la nation serbe et de ses intérêts. Aussi, après la première manifestation du 9 mars, au cours de laquelle les étudiants réclament la libéralisation politique et où l'on pense être en présence du premier mouvement de contestation politique d'envergure, l'opposition est amenée par le parti au pouvoir à capter à son profit les revendications étudiantes pour les engager dans un jeu de "surenchère" nationaliste. Lorsque Vuk Draskovic est libéré de prison, il déclare "Je ne pardonnerai jamais que le sang serbe ait coulé $(\Sigma)$ Seule une Serbie démocratique pourra défendre tous les Serbes (...) Ce n'est pas parce qu'ils m'ont relâché qu'il faut rentrer chez soi" et il s'exclame "Liberté pour la Serbie !". De toute évidence, si l'on parle encore de démocratie, le processus de démocratisation doit avoir pour but ultime le renforcement de la Serbie et la protection des Serbes - notamment des Serbes "menacés" par les autres nations de la Yougoslavie. Ainsi, le rejet du néo-communisme ne peut se faire que grâce à une stratégie nationaliste s'appuyant sur les mêmes thèmes que ceux utilisés par Milosevic au cours des rassemblements de 1987-1989.

Ce jeu de "surenchère" se précise lorsque le président de la Serbie provoque un blocage constitutionnel en refusant toute légitimité à la présidence fédérale. Recourant à la traditionnelle rhétorique patriotique, Milosevic se démarque des institutions fédérales et se pose une fois encore en défenseur d'une "Serbie menacée, qui doit s'unir pour résister aux forces du mal". Pour assurer la sécurité de la Serbie, le président demande la mobilisation des réservistes de la police, ainsi que la formation urgente de forces de l'ordre supplémentaires, qui sont envoyés dans les territoires des républiques voisines où se trouvent des minorités serbes. A ce stade de la crise, le but de Milosevic est double : retrouver son image "providentielle" et renforcer sa légitimité, menacée par la révolte étudiante et la répression qui s'ensuit; régler, par la force si cela s'avère nécessaire, les contentieux avec les autres républiques yougoslaves. Un an après les 
manifestations de Belgrade, le président serbe est toujours au pouvoir ; dans la guerre qui l'oppose à la Croatie, la population serbe est longtemps restée unie sur la question de la défense à tout prix de ses intérêts, et l'opposition n'est pas parvenue à mobiliser une population suffisante pour déstabiliser sérieusement le Parti socialiste au pouvoir.

contestation pu constater qu'au cours des quelques mouvements importants de probstion de l'ordre politique en Yougoslavie, la question nationale a constitué la des années 1960-1970, la revendication nationaliste est rendue impossible par la domination de l'idéologie marxiste - qui nie l'existence même d'un problème de rivalités nationales au sein de la fédération - et par la menace de mesures répressives par le régime au pouvoir. Afin de pouvoir exprimer des revendications à caractère national, les acteurs politiques se servent de "prétextes" économiques, tels que l'introduction de la libéralisation des marchés. Au cours de la dernière décennie, il s'agit de la dynamique opposée. Une "captation nationaliste" est opérée sur tous les mouvements de contestation reposant sur des revendications économiques ou sociales, puisque ces dernières ne sont jamais assez puissantes pour mobiliser une partie importante de la société. Aussi, des mouvements de contestation qui au départ se veulent favorables à la libéralisation politique ou économique, se transforment en une lutte pour l'affirmation de l'identité nationale, pour la défense des intérêts vitaux et pour la suprématie d'une république sur les autres. En effet, le poids de la question nationale, étouffée mais jamais résolue par le régime communiste d'après-guerre, ne permet pas la mobilisation de la société autour d'autres revendications. Celles-ci ne peuvent être utilisées que comme prétextes pour enclencher un mouvement de contestation. Ceci empêche également le développement de mouvements de contestation ou de manifestations à proprement parler "yougoslaves", mais permet uniquement à des mouvements étroitement liés à et définis par, une identité nationale serbe, croate, albanaise ou autre de s'exprimer. Si les manifestations de mars 1991 à Belgrade ont un intérêt pour le chercheur, c'est parce qu'elles expriment la cristallisation du blocage politique dans l'ex-fédération yougoslave, blocage résultant du poids de la question nationale dans toutes ses républiques. En effet, les manifestants, ainsi que les membres de l'opposition, sont entraînés à se servir des arguments nationalistes déjà utilisés par les autorités communistes serbes, arguments qu'ils rejettent au départ mais qui sont les seuls à pouvoir mobiliser une opinion publique serbe fixée sur le conflit national.

Selon Guillermo O'Donnell et Philippe Schmitter ${ }^{7}$, les périodes de transition d'un régime autoritaire à un régime démocratique sont souvent caractérisées par la négociation de "pactes" entre le pouvoir autoritaire et l'opposition. Ces pactes ont pour but de régir les modalités du passage à la démocratie, en définissant la nature des échanges et des relations entre l'espace public et 1 espace privé, entre le pouvoir et la société civile. Au c ur du pacte se trouve un compromis négocié, qui permet aux divers acteurs concernés d'abandonner volontairement une partie de leurs prérogatives et de limiter leur capacité à se servir de la violence, dans le but d'instaurer une transition progressive et contrôlée vers la démocratie. Le plus souvent, la mobilisation politique qui suit la période initiale de libéralisation conduit à l'apparition de nouveaux partis politiques, ce qui rend nécessaire l'organisation d'élections, comme étape essentielle des négociations pour un nouveau pacte. En Serbie, nous avons pu constater 
effectivement l'apparition d'un grand nombre de partis politiques à la fin des années 1980. Toutefois, la campagne électorale étant presque entièrement basée sur des arguments "nationaux", utilisés par le pouvoir aussi bien que par l'opposition, les négociations en vue d'un nouveau "pacte" politique sont bloquées. Les acteurs politiques "campent" sur leurs positions et empêchent ainsi la mise en place d'une transition "gérée" vers la démocratie. Les manifestations de mars à Belgrade montrent qu'à l'évidence, il n'y a aucune possibilité de négociation entre l'opposition et les autorités serbes ces dernières n'acceptant pas de se défaire du monopole de la violence et de l'utilisation de la force dans les processus de transition. Le pouvoir serbe oblige ainsi l'opposition à avoir recours aux mêmes arguments et aux mêmes symboles pour mobiliser la société civile dans la lutte pour un changement de l'ordre politique. D'un côté comme de l'autre, l'action est par conséquent rendue inefficace et la transition ne peut véritablement avoir lieu.

En outre, cette impasse du régime autoritaire oblige les autorités au pouvoir à choisir la "fuite en avant", afin d'éviter que l'échec de la transition négociée ne remette en question leur propre légitimité. Souvent, cette tactique se traduit par l'engagement de l'état dans un confit externe. En effet, Milosevic opte pour cette solution à la suite des manifestations de mars 1991. Se sentant menacé sur son propre terrain et dans son rôle de défenseur des intérêts serbes, il choisit "d'exporter" le débat politique à l'extérieur des frontières de la Serbie et d'engager son état dans un conflit ouvert avec la Croatie. Ceci lui permet de continuer à s'appuyer sur une rhétorique exclusivement nationaliste et de neutraliser l'opposition qui, bien que se servant de cette même rhétorique, n'a pas le monopole de la violence légitime et ne peut par conséquent se mesurer au pouvoir en place, puisqu'elle ne peut traduire ses slogans en actes concrets. Ainsi, les mouvements de contestation en Yougoslavie - manifestations, grèves et émeutes n'auront servi qu'à cristalliser, jusqu'à son éclatement, la question nationale. A aucun moment de l'histoire yougoslave récente, ils n'auront été le symptôme d'une véritable remise en cause de l'ordre politique, économique ou social, uniquement celui du malaise éprouvé par la population d'un état sans nation ${ }^{8}$.

\section{NOTES}

1. En effet, la guerre introduit, pour la recherche, plusieurs obstacles concrets, tels que les difficultés de communication entre les républiques yougoslaves, l'impossibilité d'avoir accès aux documents officiels et même à la presse locale d'époque, etc.

2. Pour une étude des conflits sociaux en Yougoslavie, voir Zdravko Mlinar "Les conflits sociaux et le développement social en Yougoslavie, Revue de l'Est vol. 3/ 1972 (2).

3. Il est par ailleurs important de noter qu'à partir de 1962, le pouvoir reconnaît pour la première fois l'existence d'une crise de l'Etat yougoslave. Cette prise de conscience n'est cependant exprimée qu'au cours d'une séance secrète des organes dirigeants du Parti en mars 1962. A cette occasion, Tito, confronté à la fragmentation de l'unité gouvernementale, se demande : $\Sigma$ "Notre pays est-il encore apte à se maintenir, à ne pas 
se désintégrer, $\Sigma$ cette communauté est-elle mûre pour la vie ou non ?" (Dusan Bilandzic, "1971-1991 ; Le putsch de Karadordevo" - Danas, 3 décembre 1991).

4. La bataille la plus implante menée par les Serbes du prince luzar contre l'empire ottoman qui s est traduite par l'écrasement de l'armée serbe et qui a valeur de mythe dans l'Histoire.

5. Pour la première fois depuis 1945.

6. "Le feu maîtrisé dans la maison serbe", Ln Croix, 15 mars 1991.

7. Guillermo O'donnell, Philippe Schmitter et Laurence Whitehead eds., Transitions From Authoritarian Rule, The John Hophins University Press, Londres, 1986.

8. Le 9 mars 19J2, le Mouvement pour le renouveau serbe de V. Draskovic a rassemblé près de 50000 personnes, au cours d'une manifestation pour commémorer les événements de mars 1991. Un certain nombre de partis d'opposition serbes avaient obtenu 200000 signatures pour une pétition visant à engager une procédure de destitution du président Milosevic. Toutefois, même si le nombre de signatures requis (100 000) pour engager un débat à ce sujet au Parlement était acquis, les efforts pour renverser le parti au pouvoir n'ont pas pu aboutir. En effet, la loi stipule que deux-tiers des 250 députés du Parlement doivent voter en faveur d'une procédure de destitution a/in qu'elle soit engagée. Or, actuellement, 194 députés seulement siègent au Parlement. Par ailleurs, les médias étant toujours contrôlés à $95 \%$ par le parti au pouvoir, la "campagne" menée contre Milosevic ne peut être aussi efficace que le voudraient les partis d'opposition. Faute de programme commun et structuré, l'opposition ne représente donc pas encore, une solution de rechange possible pour la Serbie. la position de Slobodan Milosevic dépendra de l'évolution du conflit serbocroate ainsi que du conflit qui se développe entre la Serbie et la Bosnie-Herzégovine et le Kosovo.

INDEX

Index géographique : Balkans

Mots-clés : mouvements politiques, Nationalismes, résistances politiques 